\title{
Guía para la determinación de la amortización del activo biológico de acuerdo a la NIC 41
}

\author{
Fecha de recepción: 13/11/2019 • Fecha de aceptación: 02/01/2020 • Fecha de publicación:10/02/2020
}

\author{
Juan Carlos Tene \\ Avesca Avicola Ecuatoriana \\ juan199206@hotmail.com \\ https://orcid.org/0000-0003-2348-0919
}

\section{Resumen}

El Activo Biológico dentro de la empresa productiva es uno de los pilares fundamentales al momento de analizar la razonabilidad del proceso de amortización que tendrá el activo frente a la producción obtenida durante un periodo. En la actualidad implementar un cambio dentro de un proceso ya establecido genera grandes contradicciones, porque imperan análisis de procesos, optimización de resultados y control de actividades que ayuden a obtener mejoras a nivel financiero. El objetivo del presente artículo es analizar el proceso para el cálculo de la amortización de los activos bilógicos bajo normativa "Normas Internacionales de Contabilidad NIC 41". En cuanto al método de investigación, es de tipo documental con un alcance descriptivo, bajo el método deductivo. La fuente de información para el tratamiento de los datos es una empresa productiva del sector avícola, permitiendo conocer los pasos para obtener el valor amortizable por mes de los lotes productivos de la empresa de análisis. La principal conclusión es que los pasos presentados para llegar al valor que posteriormente será registrado contablemente soportan de forma clara el tratamiento del activo biológico en toda su etapa de producción, aunque el cálculo para determinar el valor permite analizar el costo de amortización por unidades totales producidas durante el mes, es necesario recalcar que el importe en libros se verá reflejado durante el costeo del huevo durante el mes de cierre en la cuenta Inventarios. 


\begin{abstract}
The Biological Asset within the productive company is one of the fundamental pillars when analyzing the reasonableness of the amortization process that will have the active front to the production obtained during a period. At present, implementing a change within an established process generates great contradictions, because process analysis, results optimization and control of activities that help to obtain improvements at the financial level prevail. The objective of this article is to analyze the process for the calculation of the amortization of the biological assets under the norm "International Accounting Standards IAS 41".

As for the research method, it is of documentary type with a descriptive scope, under the deductive method. The source of information for the treatment of the data is a productive company of the poultry sector, to know the steps to obtain the amortizable value per month of the productive lots of the analysis company.

The main conclusion is that the steps established to arrive at the value that will subsequently be recorded in a clearly accountable way the treatment of the biological asset throughout its production stage, although the calculation to determine the value allows analyzing the amortization cost per total units produced During the month, it is necessary to emphasize that the amount in books will be reflected during the cost of the egg during the closing month in the Inventory account.
\end{abstract}

KEYWORDS: Biological assets, reasonableness, amortization, NIC 41. 


\section{Introducción}

Las empresas en la actualidad serán capaces de sobresalir y coexistir, si tienen una determinación clara en sus costos en cada periodo de cierre, esto genera un llamado de atención a los profesionales que carecen de un conocimiento acerca del cálculo de la Amortización en los Activos Biológicos en las organizaciones enfocadas a una actividad productiva. Si se puede llegar a optimizar los costos dentro del proceso generará grandes resultados al momento de analizar los estados de Pérdidas y Ganancias por cada línea de negocio. El objetivo principal de este artículo es desarrollar una guía para la determinación de la amortización de los activos biológicos que se manejan dentro de las empresas.

En cada proceso de análisis que se presenta en los centros productivos se ve inmerso un punto primordial, la amortización del biológico en las actividades avícolas, los costos se conocen posterior al cuidado y desarrollo de las transformaciones biológicas de los animales. Es justamente por medio de este proceso que los pasos a seguir para llegar a presentar una información que sirva de soporte al objetivo planteado anteriormente será 1.- Examinar las Normas Internacionales de Contabilidad NIC 41 respecto al manejo y tratamiento de los activos biológicos, 2.- Presentar la información que sirva de soporte para el realizar el proceso de cálculo y 3.- Determinar la amortización del activo biológico paso a paso.

Para el proceso de investigación se consideró las siguientes definiciones, en vista que durante el material presentado se observará a detalle la terminología empleada.

\section{Normas Internacionales de Contabilidad NIC 41 Agricultura}

Cada empresa debe establecer las Normas Internacionales de Información Financiera NIIF que le son aplicables en el tratamiento contable correspondiente. En el caso específico de las empresas que se dedican a la actividad agrícola y que cumplen con el principio fundamental de accountability pública, además de que no se enmarcan en las condiciones de Normas Internacionales de Información Financiera para Pequeñas y Medianas Entidades NIIF para PYMES, deben aplicar NIIF plenas bajo la Norma Internacional de Contabilidad 41: AGRICULTURA.

El término AGRICULTURA o AGRICULTURE en NIIF / IFRS se refiere al uso de la tierra para cultivo y cría animales para proporcionar alimentos y otros productos. Así como también Agricultura Activity que significa Actividad Agrícola; y Biological porque se trata de organismos vivos. De acuerdo a las NIc 41 (2014) se establece que:

La NIC 41 prescribe, entre otras cosas, el tratamiento contable de los activos biológicos a lo largo del periodo de crecimiento, degradación, producción y procreación, así como de la medición inicial de los productos agrícolas en el punto de su cosecha o recolección

La NIC 41 no se ocupa del procesamiento de los productos agrícolas tras la recolección de la cosecha; por ejemplo no trata del procesamiento de las uvas para obtener vino, ni el de la lana para obtener hilo. 
La NIC 41 exige que los cambios en el valor razonable menos los costos de venta de los activos biológicos sean incluidos como parte de la ganancia o pérdida del periodo en que tengan lugar.

El objetivo de esta Norma es prescribir el tratamiento contable, la presentación en los estados financieros y la información a revelar en relación con la actividad agrícola.

La entidad reconocerá un activo biológico o un producto agrícola cuando, y sólo cuando:

(a) la entidad controle el activo como resultado de sucesos pasados;

(b) sea probable que fluyan a la entidad beneficios económicos futuros asociados con el activo;

(c) el valor razonable o el costo del activo puedan ser medidos de forma fiable.

Las NIC 41 hacen un aporte relevante en el tema de activos biológicos, ya que la contabilidad tradicional solo se basa en el coste histórico y en el principio de realización, se deberá tener en cuenta el concepto valor justo como medio de valoración, es decir el activo biológico tiene que ser medido con base en el momento de reconocimiento inicial y se vuelve a medir cada año, es ahí en la variación que se genera la diferencia. Arévalo E., Pulido D., Rangel A (2017).

\section{Enfoque Productivo}

\section{Actividad agrícola}

Es la gestión, por parte de una entidad, de la transformación biológica y recolección de activos biológicos, para destinarlos a la venta, o para convertirlos en productos agrícolas o en otros activos biológicos adicionales. Se considera Activo Biológico, como un animal vivo o una planta.

\section{Transformación biológica}

Comprende los procesos de crecimiento, degradación, producción y procreación que son la causa de los cambios cualitativos o cuantitativos en los activos biológicos.

Previo al proceso de tratamiento biológico de las aves se realizan los trámites financieros en función de adquirir el lote para la empresa, en este caso se obtienen los permisos aduaneros, las negociaciones con los proveedores internacionales, la generación de los documentos soporte (Facturas, Retenciones, Aprobación Agrocalidad).

En todo proceso productivo, se consideran mínimo 3 componentes en los costos: como son materia prima (MP), mano de obra directa (MOD) y costos indirectos de fabricación (CIF). Siendo estos últimos conformados por mano de obra indirecta, alquiler, servicios, etc. Polimeni, R. y otros (1997).

En el caso de la producción avícola, luego de realizados todos estos procesos llegan las aves al país y son trasladadas a la granja donde iniciará el proceso. Según Verdesoto (2014) las etapas de este proceso son: 
- Etapa Levante

Conocida como la crianza de las aves, en este proceso las aves son distribuidas por galpones dentro de estas instalaciones son cuidadas, alimentadas y vacunadas durante 24 semanas (6 meses), tiempo suficiente para que el ave adquiera peso y la condición adecuada para que realice la etapa de apareamiento.

Durante las 24 semanas los costos en los que incurre la Granja que son Mano de Obra, (MOD) Materia Prima (MP), y Costos Indirectos de Fabricación (CIF) son acumulados por cada mes en una cuenta denominada "Costo Histórico Animales Vivos en Crecimiento", esta cuenta es de acumulación hasta llegar al sexto mes donde inicia la etapa productiva del Activo Biológico

\section{- Etapa Producción}

En este escenario las aves están listas para realizar el proceso productivo, el mismo que se asocia a la puesta de Huevo Fértil, el cual es recolectado por los galponeros durante el día, por 4 ocasiones para llegar a una recolección final del Huevo fértil durante los 30 días del mes se realiza esta actividad. Hay que tomar en cuenta que las 24 semanas pueden empezar a inicios de mes y para llegar a calcular la amortización se debe esperar que se cierre el mes donde el lote inicio producción llegando a tener al cierre de mes una edad de 28 a 29 semanas dependiendo el día cuando inicio etapa productiva.

Esta información que se adquiere por el aspecto productivo del lote de aves, es la parte principal dentro del cálculo de la Amortización del Activo Biológico ya que toma como base la cantidad producida del mes frente a la proyección de la producción ideal del lote.

Cuando se obtiene el valor de la Amortización, contablemente juega dos cuentas la primera que ingresa a la cuenta denominada Amortización de Activos Biológicos, y la segunda que se registra a la cuenta denominada Deterioro de Animales Vivos en Producción de esta forma se va disminuyendo el valor acumulado contablemente del Activo durante las 24 semanas en las que estuvo el lote de aves en crecimiento

\section{- Etapa Cierre}

En esta parte el lote de producción ha cumplido 36 semanas de producción después de la semana 24, llegando a tener una edad biológica de 60 semanas, bajo este antecedente el lote esta de salida el valor residual para cada raza de aves difiere en función de su genética y por decisión de gerencia. Para esto el valor de rescate llega a ser de aproximadamente $\$ 5.00$ por ave.

Permitiendo vender el lote de aves viejas ya que su ciclo productivo por la edad en la que se encuentran ha culminado. Para el lapso de venta de las aves se presenta diferentes condiciones de mercado, para esto la gerencia destina un tiempo estimado de 2 semanas más a las 60 semanas que ya posee el ave, en este tiempo el área comercial debe colocar las aves viejas al mercado para venderlas, culminando la vida del lote con una edad de 62 semanas. 


\section{- Clasificación de la Producción}

Dentro de la granja cuando esta entra en etapa productiva, se presenta dos escenarios en el primero se aprecia la recolección de los huevos en cada uno de los galpones que posea la granja, en esta actividad el galponero debe tomar los huevos que hayan puestos las aves de forma inmediata y llevarlas a la zona de clasificación, cuando los huevos llegan a esta parte las personas encargadas de este sector reciben los huevos y proceden a lavar el producto, finalmente lo clasifican por tipo de huevo en este caso pueden ser Huevos Fértiles destinados a ser incubados para luego obtener un producto adicional y posteriormente venderlo, otra clasificación es el Huevo Comercial destinado para consumo humano el cual es vendido a clientes y finalmente el Huevo Roto, que por manipulación en el trasporte o en la recepción el huevo pierde su condición física (Cascara del Huevo se rompe) dejando así al huevo no apto ni para el consumo ni para incubarlo por ende lo desechan, es el denominado Compostera. (Avesca, 2019)

\section{MÉTODOS Y MATERIALES}

La investigación que se va a desarrollar es una investigación documental con un alcance descriptivo y bajo el método deductivo, ya que se procura obtener información que sirva de soporte para el tratamiento de los datos. La fuente de información son datos de la empresa AVESCA AVICOLA ECUATORIANA C.A, específicamente de la granja Cuendina, considerando en particular el lote 549, en el cual se adquieren aves, tanto hembras como machos. Se obtienen tablas con los datos pertinentes, en las cuales se realizan los cálculos que derivan paso a paso en el monto de la amortización de estos activos biológicos.

\section{RESULTADOS}

AVESCA AVICOLA ECUATORIANA C.A es una empresa que basa su economía en la crianza y producción de aves de corral, además de comercializar productos encaminados a la fortificación del suelo agrícola del sector. (Avesca, 2019)

De esta forma la actividad productiva cobra valor dentro del estudio planteado ya que se abordarán las prácticas que conllevan a manejar el activo biológico desde el momento de su ingreso a las granjas de la empresa. La información que se describe a continuación es una muestra del tratamiento que se brinda a los lotes productivos dentro de la empresa. Para la investigación se consideran los datos de la Granja Cuendina, específicamente el lote 549.

Cuadro 1

Costo inicial. Septiembre 2018

\begin{tabular}{|c|c|c|c|c|c|c|c|c|c|c|c|}
\hline \multirow{2}{*}{$\#$} & \multirow{2}{*}{ RAZA } & \multirow{2}{*}{ GENERO } & \multirow{2}{*}{ LOTE } & \multicolumn{2}{|c|}{ INGE SOS PERODO } & \multirow{2}{*}{$\begin{array}{l}\text { CARGO } \\
\text { ALIMENTO }\end{array}$} & \multirow{2}{*}{$\begin{array}{r}\text { CARGO } \\
\text { M.O.D }\end{array}$} & \multirow{2}{*}{$\begin{array}{l}\text { CARGO } \\
\text { GIF. }\end{array}$} & \multicolumn{2}{|c|}{ DSPON BLLE PERODOO } & \multirow{2}{*}{$\begin{array}{l}\text { COSTO } \\
\text { UNTARO }\end{array}$} \\
\hline & & & & CANT. & VALOR & & & & CANT. & VALOR & \\
\hline 1 & $\operatorname{COBB}$ & HEMBRAS & 549 & 31,143 & $183,394.28$ & $6,926.99$ & $18,013.66$ & $37,358.39$ & 31,143 & $245,693.31$ & 7.89 \\
\hline 1 & $\operatorname{COBB}$ & MACHOS & 549 & 4,686 & $42,836.38$ & $1,042.28$ & $2,710.46$ & $5,621.21$ & 4,686 & $52,210.34$ & 11.14 \\
\hline TOTA & & & & $35,829.00$ & $226,230.66$ & $7,969.27$ & $20,724.12$ & $42,979,60$ & $35,829.00$ & $297,903.65$ & \\
\hline
\end{tabular}


Dentro del cuadro presentado se aprecia la forma de acumulación del nuevo lote que ingresa a etapa de levante, en el mismo se presenta el valor por el costo de la Importación realizada, adicional a esto se desglosa el detalle de los valores a acumular dentro del primer mes de ingreso, de esta forma se va a cumulando el activo biológico hasta llegar a semana 24, cabe recalcar que el número de aves encastadas al inicio del lote cambiará hasta la semana 24 en vista que la diferencia se origina por concepto de mortalidad de las aves que se puedan presentar en ese tiempo.

Por ende hay que tener claro que el inventario de aves al inicio no puede ser igual al momento de que el lote pase a etapa productiva, en esa etapa se considera como inventario inicial el saldo final de aves que hayan llegado hasta inicios de la semana 24, por esta razón es importante considerar esta variación ya que es el manejo normal que lleva a cabo el cálculo regular dentro de los datos adquiridos por parte de la empresa.

Cuadro 2.

Producción Marzo de 2019

\begin{tabular}{|r|r|r|r|r|}
\hline Fecha & Comerciales & Compostera & Fértiles & Total \\
\hline $07 / 03 / 2019$ & $1.287,00$ & 8,00 & & $1.295,00$ \\
\hline $08 / 03 / 2019$ & 827,00 & 3,00 & & 830,00 \\
\hline $09 / 03 / 2019$ & $1.170,00$ & 18,00 & & $1.188,00$ \\
\hline $10 / 03 / 2019$ & $1.748,00$ & 9,00 & & $1.757,00$ \\
\hline $11 / 03 / 2019$ & $1.377,00$ & 36,00 & 884,00 & $2.297,00$ \\
\hline $12 / 03 / 2019$ & & $1.944,00$ & $1.182,00$ & $3.126,00$ \\
\hline $13 / 03 / 2019$ & & $2.613,00$ & $1.457,00$ & $4.070,00$ \\
\hline $14 / 03 / 2019$ & & $1.362,00$ & $3.772,00$ & $5.134,00$ \\
\hline $15 / 03 / 2019$ & & $1.190,00$ & $5.346,00$ & $6.536,00$ \\
\hline $16 / 03 / 2019$ & & $1.482,00$ & $6.304,00$ & $7.786,00$ \\
\hline $17 / 03 / 2019$ & & $1.515,00$ & $7.709,00$ & $9.224,00$ \\
\hline $18 / 03 / 2019$ & & $1.537,00$ & $9.338,00$ & $10.875,00$ \\
\hline $19 / 03 / 2019$ & & $1.206,00$ & $10.906,00$ & $12.112,00$ \\
\hline $20 / 03 / 2019$ & & $1.277,00$ & $9.344,00$ & $10.621,00$ \\
\hline $21 / 03 / 2019$ & & $2.384,00$ & $16.846,00$ & $19.230,00$ \\
\hline $22 / 03 / 2019$ & & $1.535,00$ & $15.140,00$ & $16.675,00$ \\
\hline $23 / 03 / 2019$ & & $1.459,00$ & $16.299,00$ & $17.758,00$ \\
\hline $24 / 03 / 2019$ & & $3.866,00$ & $14.599,00$ & $18.465,00$ \\
\hline $25 / 03 / 2019$ & $3.455,00$ & 158,00 & $16.084,00$ & $19.697,00$ \\
\hline $26 / 03 / 2019$ & $2.941,00$ & 256,00 & $17.220,00$ & $20.417,00$ \\
\hline $27 / 03 / 2019$ & $3.051,00$ & 162,00 & $17.413,00$ & $20.626,00$ \\
\hline $28 / 03 / 2019$ & $3.362,00$ & 192,00 & $17.787,00$ & $21.341,00$ \\
\hline $29 / 03 / 2019$ & $2.919,00$ & 236,00 & $18.229,00$ & $21.384,00$ \\
\hline $30 / 03 / 2019$ & 887,00 & 217,00 & $20.919,00$ & $22.023,00$ \\
\hline $31 / 03 / 2019$ & 968,00 & 264,00 & $21.443,00$ & $22.675,00$ \\
\hline & $\mathbf{2 3 . 9 9 2 , 0 0}$ & $\mathbf{2 4 . 9 2 9 , 0 0}$ & $\mathbf{2 4 8 . 2 2 1 , 0 0}$ & $\mathbf{2 9 7 . 1 4 2 , 0 0}$ \\
\hline \hline Totalgeneral & & & & \\
\hline
\end{tabular}


En este cuadro se aprecia el detalle de las producciones por día dentro del mes donde inicia producción el lote que ingreso 24 semanas atrás a la Granja Cuendina, adicional a esto se aprecia como las producciones van creciendo en función de los días. Hay que considerar que las producciones de los lotes productivos se encuentran en función de la semana biológica del ave por ende no necesariamente debe llegar a producir desde el primer día del mes, el ejemplo demuestra que el lote ingresa en producción en el mes de Marzo del año 2019 pero a partir del día 7 del mes ya que biológicamente el ave en este día empezó a poner huevos.

\section{Determinación del Cálculo de la Amortización}

\section{Paso 1: Obtención de Datos de Acumulación del Activo Biológico}

Por medio de los datos tomados de la empresa, se procedió a realizar un cuadro resumen de los meses en los que el lote de aves se encontró en proceso de crecimiento identificando cada uno de los elementos del costo que fueron acumulados durante este tiempo en la cuenta Costo Histórico Animales Vivos en Crecimiento. El cálculo es al costo inicial de $\$ 297.903,65$, se suman todos los costos de Mano de obra directa, materia prima y costos indirectos de fabricación en que se incurrieron durante las 24 semanas de periodo de levante

\section{Cuadro 3}

Resumen del levante,Septiembre 2018- marzo 2019

\begin{tabular}{|r|c|c|c|c|c|c|}
\hline FECHA & COMPRA \$ & MP \$ & MOD \$ & CF \$ & NFF & TOTAL \\
\hline sep-18 & $226.230,66$ & $7.969,27$ & $20.724,12$ & $42.979,60$ & - & $297.903,65$ \\
\hline oct-18 & - & $24.373,96$ & - & $24.742,30$ & - & $347.019,91$ \\
\hline nov-18 & - & $24.203,67$ & $7.295,27$ & $25.902,24$ & - & $404.421,09$ \\
\hline dic-18 & - & $28.438,90$ & $2.334,71$ & $20.727,37$ & - & $455.922,07$ \\
\hline ene-19 & - & $39.651,67$ & $6.161,58$ & $28.726,69$ & - & $530.462,01$ \\
\hline feb-19 & - & $46.494,67$ & $8.506,40$ & $16.589,54$ & - & $602.052,62$ \\
\hline mar-19 & - & $1.617,80$ & - & 881,54 & - & $604.551,96$ \\
\hline
\end{tabular}

Paso 2: Identificación del Saldo Inicial de las Aves en Producción

Para obtener esta información se procedió a bajar los inventarios finales del último mes en el que el lote de aves estuvo en proceso de levante ya que se convertirá en el inventario inicial del nuevo mes, en donde el lote inicia producción. El cálculo se obtiene restando al inventario inicial de 31.143 aves hembras, el número de aves que no comenzaron el proceso de producción de huevos. 
Cuadro 4

Inventario inicial de producción, Marzo 2019

\begin{tabular}{|c|c|c|c|c|c|c|}
\hline \multirow{2}{*}{ GRANJA } & \multirow{2}{*}{ \# } & \multirow{2}{*}{ RAZA } & \multirow{2}{*}{ GENERO } & \multirow{2}{*}{ LOTE } & \multicolumn{2}{|c|}{ INVENTARIO INICIAL } \\
\hline & & & & & CANT. & VALOR \\
\hline \multirow[t]{2}{*}{ CUENDINA } & 1 & COBB & HEMBRAS & 549 & 29.183 & $604.551,96$ \\
\hline & 1 & $\mathrm{COBB}$ & MACHOS & 549 & 3.223 & 0,00 \\
\hline
\end{tabular}

\section{Paso 3: Cálculo de la Producción Ideal del lote}

Este cálculo se lo obtiene al multiplicar la cantidad de huevos producidos diariamente durante la vida biológica del ave (propuesto por el área técnica, es un coeficiente variable) por el saldo de vida útil de las aves (33 semanas por 7 días son 231 días) por el número de aves que realmente iniciaron la producción. (29.183 aves)

Cuadro 5

Producción ideal, Marzo 2019

\begin{tabular}{|c|c|c|c|c|c|c|c|c|c|c|c|}
\hline \multirow{2}{*}{ GRANA } & \multirow{2}{*}{ i } & \multirow{2}{*}{ ROLA } & \multirow{2}{*}{ GENERO } & \multirow{2}{*}{ LOTE } & \multicolumn{2}{|c|}{ INESTAROO NCALL } & \multirow{2}{*}{$\begin{array}{l}\text { U.Y. } \\
\text { CANT. }\end{array}$} & \multirow{2}{*}{$\begin{array}{l}\text { EADFA } \\
\text { MAQZ2O }\end{array}$} & \multirow{2}{*}{$\begin{array}{l}v_{0.1} \\
550 .\end{array}$} & \multirow{2}{*}{$\frac{\mathrm{NES} .}{\mathrm{Vill}}$} & \multirow{2}{*}{$\begin{array}{l}\text { PROOUD. } \\
\text { DEAL }\end{array}$} \\
\hline & & & & & CANT. & VALOR & & & & & \\
\hline CUENONA & 1 & $C O B B$ & HEVBRAS & 548 & 29.183 & $604.551,00$ & 3355 & $\theta$ & 62 & & 4.690 .091 \\
\hline & 1 & $C O B B$ & WACHOS & 549 & 3223 & 0,00 & 170 & $\theta$ & 62 & & \\
\hline
\end{tabular}

\section{Paso 4: Obtención de la Producción Real del mes}

Para tener estos datos se procedió a bajar del sistema la información concerniente de la producción del lote 549 que tuvo su inicio en el mes de Marzo del 2019, los datos fueron recolectados en su totalidad por cada clase de Huevo es decir, Huevo Fértil, Huevo Comercial y Huevo Roto.(Compostera)

Cuadro 6

Producción real mensual, Marzo 2019

\begin{tabular}{|r|r|r|r|c|}
\hline Fecha & Comerciales & Compostera & Fértiles & Total \\
\hline Total general & $\mathbf{2 3 . 9 9 2 , 0 0}$ & $\mathbf{2 4 . 9 2 9 , 0 0}$ & $\mathbf{2 4 8 . 2 2 1 , 0 0}$ & $\mathbf{2 9 7 . 1 4 2 , 0 0}$ \\
\hline
\end{tabular}




\section{Paso 5: Identificación del Valor Residual para el Lote}

De acuerdo a lo que menciona la NIC 41 la empresa en función de las condiciones del mercado puede establecer un valor de rescate que debe diferenciarse del valor razonable como tal, dentro de la investigación que se realizó la empresa realiza un análisis del mercado y a través de este genera un valor residual del lote al término de su vida biológica que es de $\$ 5.00$ a este costo saldría el ave una vez que culmine su etapa productiva y salga a la venta. El cálculo se realiza restando al Inventario inicial de aves (29.183) las aves que se estiman que no terminan la etapa de producción (335), lo que da como resultado 28.848 , cantidad que se multiplica por el costo unitario de $\$ 5$.

Cuadro 7

Valor residual de aves por lote, Marzo 2019

\begin{tabular}{|c|c|c|c|c|c|c|c|c|}
\hline \multirow{2}{*}{$\begin{array}{l}\text { NOMBRE } \\
\text { GRANJA }\end{array}$} & \multirow{2}{*}{$\#$} & \multirow{2}{*}{ RAZA } & \multirow{2}{*}{ GENERO } & \multirow{2}{*}{ LOTE } & \multicolumn{2}{|c|}{ INVENTARIO INICIAL } & \multirow{2}{*}{$\begin{array}{l}\text { M.Y. T. } \\
\text { CANT. }\end{array}$} & \multirow{2}{*}{$\begin{array}{c}\text { VALOR } \\
\text { RESIDUAL }\end{array}$} \\
\hline & & & & & CANT. & VALOR & & \\
\hline CUENDINA & 1 & COBB & HEMBRAS & 549 & 29.183 & $604.551,96$ & 335 & $144.240,00$ \\
\hline & 1 & $\cos B$ & MACHOS & 549 & 3.223 & 0,00 & 170) & 0,00 \\
\hline
\end{tabular}

\section{Paso 6: Cálculo de la Amortización}

El cálculo de la amortización se realiza en función de los pasos anteriores de la siguiente forma, se toma la diferencia del Valor Total Acumulado $(\$ 604$. 551,96) menos el Valor de Rescate del lote (\$144.240) a este resultado se le denomina Saldo a Amortizar. Luego a este Saldo se le divide para la cantidad de huevos que le faltan por producir al lote que entra a producción (4.690.091 huevos) y con el coeficiente que se obtenga se multiplica por la cantidad total de huevos que produjo en el mes. (297.142 huevos)

Cuadro 8

Amortización de aves por lote, Marzo 2019

\begin{tabular}{|c|c|c|c|c|c|c|c|c|c|c|c|c|c|}
\hline GRANA & t & RAZA & GENERO & LOTE & $\frac{\text { ARO NICLAL }}{\text { VALOR }}$ & $\begin{array}{l}\text { M.YT. } \\
\text { CAST. }\end{array}$ & $\begin{array}{l}\text { EQAD FII } \\
\text { MARZO }\end{array}$ & $\begin{array}{c}\text { PROOWOL. } \\
\text { IDEAL }\end{array}$ & $\begin{array}{l}\text { SALDOXX } \\
\text { PAOOUUR }\end{array}$ & $\begin{array}{l}\text { PRODOUD. } \\
\text { MES }\end{array}$ & $\begin{array}{l}\text { VALOR } \\
\text { PESSOUAL }\end{array}$ & $\begin{array}{l}\text { SALDOA } \\
\text { AMOQTIL }\end{array}$ & $\begin{array}{l}\text { AMORTRL } \\
\text { HENRAS }\end{array}$ \\
\hline CUENDINA & 1 & $\mathrm{COBA}$ & HEYBRAS & 549 & $604.551,98$ & 335 & 28 & 4.690 .091 & 4.690091 & 297.142 & $144.240,00$ & 460.311.96 & 29.16319 \\
\hline & 1 & $C O B 8$ & MACHOS & 49 & 0,00 & 170 & 28 & & & & 0,00 & & \\
\hline
\end{tabular}




\section{DISCUSIÓN}

Al cotejar los datos de acumulación del lote se puede apreciar que el cuadro resumen con los costos obtenidos para este lote durante las 24 semanas en las que estuvo en etapa de crecimiento afloro un costo unitario por ave de $\$ 20.72$. Con este costo de acumulación por ave arranca etapa productiva este lote.

También se puede apreciar que de la producción que se presentó en el mes de Marzo el 83.54\% pertenece a Huevo Fértil, mientras que el $8.07 \%$ y el $8.39 \%$ pertenecen al Huevo Comercial y al Huevo Roto (compostera) respectivamente. Esta información demuestra que la producción de arranque del lote es beneficiosa en su primer mes de producción.

El cálculo de la producción ideal, está supeditado a un coeficiente estimado por el área técnica que determina el número de huevos esperado por cada ave al día, (que en este caso es muy conservador, puesto que resulta en promedio menos de un huevo por día) para así calcular los huevos que faltan por producir por las aves en etapa de producción (29.183 aves) en las semanas que restan de vida útil (que son en total 231 días)

\section{CONCLUSIONES}

Respecto al Marco jurídico la normativa de la NIC 41 establece que una empresa puede reconocer un activo biológico, cuando y solo cuando invierte recursos de la empresa para precautelar el activo llegando a obtener un beneficio futuro dentro de un lapso de tiempo, la empresa investigada cumple con este parámetro para que se reconozca al activo biológico como tal

Al analizar el proceso de cálculo de la amortización del activo biológico se concluye que los pasos presentados para llegar al valor que posteriormente será registrado contablemente soportan de forma clara el tratamiento del activo biológico en toda su etapa de producción, si bien es cierto el cálculo para llegar al valor permite analizar el costo de amortización por unidades totales producidas durante el mes, es necesario recalcar que el importe en libros se verá reflejado durante el costeo del huevo durante el mes de cierre en la cuenta Inventarios, ya que el Activo Biológico disminuye mes a mes por efecto de la amortización. Adicional a esto se presume que el tratamiento para el valor razonable será siempre bajo análisis y condiciones del mercado, permitiendo de esta forma cambiar el valor por ave en las diferentes situaciones que se presentar durante la etapa productiva del lote.

Finalmente, seria optimo que se pueda trabajar bajo una cantidad estándar para producción diaria de Huevo / Ave, al momento de calcular la producción ideal de los nuevos lotes que entren a producción, ya que toma tiempo llegar al coeficiente de producción de Huevo / Ave esperando los datos proporcionados por el área técnica. 


\section{REFERENCIAS BIBLIOGRÁFICAS}

Verdesoto Reinoso, M., \& Vargas Jiménez, M. (2015). Introducción a la Contabilidad Agropecuaria. (U. T. Machal, Ed.) Ecuador: Ediciones utmach.

NIC 41 Agricultura (2014). Norma Internacional Contable Agricultura. Recuperado de:file://C:/Users/Usuario/ Desktop/U_ISRAEL/10_Dec_Semestre/Tall_Competencias/Portafolio/Art_Cient_/ias41\%20Agricultura. pdf

Arévalo E., Pulido D., Rangel A (2017). La Amortización Contable de los Activos Biológicos. Recuperado de: http://revistas.sena.edu.co/index.php/finn/article/view/1497/1671

Avesca (2019). Base datos de la empresa. Recuperado de: https://www.avesca.com.ec/

Polimeni R, Fabozzi F, Adelberg A, (1997). Contabilidad De Costos. Tercera Edicion Bogota, Colombia: Mc Graw Hill 